\title{
Prospect of Manufacturing and Design Based on Physiological Polymorphism
}

\author{
Masashi Nakamura ${ }^{1)}$, Teruhiko Fuwa ${ }^{2)}$, Kaoru Inoue ${ }^{3)}$, Fusako Iwasaki ${ }^{4)}$, Susumu Kudo ${ }^{5)}$, \\ Hideki Sako $^{6}$, Masahiko Sato ${ }^{7)}$ and Yoshihiro Shimomura ${ }^{8)}$ \\ 1) Kyoto University \\ 2) Polytechnic University \\ 3) Hokkaido University \\ 4) Bunka Women's University \\ 5) Shibaura Institute of Technology \\ 6) Shizuoka University of Art and Culture \\ 7) Nagasaki Junior College \\ 8) Chiba University
}

\begin{abstract}
Modern manufacturing and design should satisfy not only the requirements of high cost performance but also of the user. Besides that, the social environment which surrounds manufacturing is rapidly changing depending on new technologies. To create future products with user satisfaction, the effective use of human physiological data is essential. This is where knowledge of physiological anthropology can be applied. Physiological anthropologists have been pointing out a limit to the interpretation of the physiological data based on its average value. They have begun to notice that the physiological functions of humans show various types according to the blended effect of heredity and the surroundings. Adequate consideration of physiological polymorphism is indispensable to accomplish manufacturing that is well devised for human. In this study the concept of manufacturing and design based on physiological polymorphism is expressed. The target and the methodology for new manufacturing are discussed in seven fields, that is, welfare equipment, clothes, artificial tissue, sporting gear, furniture, building materials, and human interface. Through the above discussion, a procedure to achieve manufacturing and design based on physiological polymorphism is proposed. J Physiol Anthropol 26(4): 507511, $2007 \mathrm{http}: / / w w w . j s t a g e . j s t . g o . j p /$ browse/jpa2

[DOI: 10.2114/jpa2.26.507]
\end{abstract}

Keywords: physiological polymorphism, manufacturing, welfare equipment, clothes, artificial tissue, sporting gear, furniture, building materials, human interface

\section{Introduction}

Modern manufacturing and design should satisfy not only the requirements of high cost performance but also the user. With that background there is a change in the aim of manufacturing. According to Iguchi (1994), in the period of rapid economic growth in Japan (1955-1973), Japanese people or society preferred "heavy, thick, long, and large" products and emphasized the merit of mass production. In the next 20 years Japanese manufacturing shifted to a "light, thin, short, and small" mode with the progress of electronics. In this period, the Japanese sense of values switched from "quantity" to "quality." Thereafter, their pursuit of quality has diversified and their manufacturing has moved to a "beautiful, amusing, creative, and sensitive" mode which pays more attention to the individual user. Besides that, the social environment which surrounds manufacturing is rapidly changing, depending on new technologies like the Internet.

In such a situation, Shimizu (1996) proposed a new "consumer model" which was the manufacturing model based on the view of users. The consumer model consisted of three parts: the "physical model," the "psychological model," and the "physiological model."

The physical model tries to make it clear which physical properties (hardness, stiffness, roughness, reflection, shape, and so on) affect the psychological or physiological evaluation of the products. This model also includes the numerical modeling of the human body and the nervous system to investigate the usability of the products.

The psychological model aims to describe what stimulus the environment gives humans and what feelings will be evoked by 
it. Various psychological indicators which reflect human mental states are tested in order to create a useful mental model. In terms of fundamental methodology, this model considers that it is "comfortable (pleasant)" when the condition of $\partial S / \partial t>0$ applies and it is "uncomfortable (unpleasant)" when the condition of $\partial S / \partial t<0$ applies, where $S$ is a mental state related to "comfortable or uncomfortable (pleasant or unpleasant)," and $t$ is time.

The physiological model emphasizes the formulation of the individual physiological characteristics. For example, changes in skin temperature or muscular activity when a user grips the handle of a certain product may be measured. The autonomic and central nervous system activity which corresponds to the change of the feeling or affection also may be applied to optimize the model. However, as the fundamental methodology, this model evaluates the human physiological state as "comfort (pleasantness)" when the condition of $\partial D / \partial t \cong 0$ occurs, where $D$ is a physiological stress related to "comfortable or uncomfortable (pleasant or unpleasant)."

One of the difficult problems in the above physiological model is that no concrete method or clear criteria to evaluate the physiological data appropriately are indicated. However, the most important thing pointed out in this model is that to create future products with high user satisfaction, the effective use of human physiological data is essential. This is where knowledge of physiological anthropology can be applied.

\section{Manufacturing and Physiological Anthropology}

Physiological anthropology is not aimed at manufacturing. Physiological anthropology aims to understand the human as a biological, social, and cultural being who is alive at the present age. This field investigates the mechanism of the exquisite adaptation and the potential physiological function of the human. Researchers in this field always feel through daily experiment that individual differences are reflected in the various physiological data they obtain. And they also know that the variations in the physiological data from the same subject are large because the human is always in a state of transition. Therefore, the researchers have pointed out the limits of the interpretation of physiological data based on its average value.

Actually, manufacturing that considers each user has been carried out already, and many products in accordance with this consideration have been marketed. Maybe the designer or manufacturer of these products would have taken account of the individual differences of age, gender, body size, preference, and so on. But these criteria are probably too simple and plain to classify human beings.

On the other hand, researchers in the field of physiological anthropology report that individual differences in the physiological response provoked by a certain stimulus can be classified into a number of types (Iwanaga et al., 2005; Maeda, 2005; Miyazaki and Tsunetsugu, 2005; Sato, 2005; Yasukouchi, 2005; Takagi et al., 2006). In other words, they have begun to notice that the physiological functions of humans show vary according to the blended effect of heredity and the surroundings. Adequate consideration for physiological polymorphism (or polytypism) is indispensable to accomplish manufacturing that is well devised for human. The authors' proposal here is for manufacturing and design based on physiological polymorphism; it is manufacturing that seriously considers differences among the types of human physiological functions.

\section{Manufacturing and Design Based on Physiological Polymorphism}

A great deal of manufacturing has recently been linked with information technology. For instance, mobile phone models change every six months, and slim-type television systems are becoming larger and larger every month. Such progressive products advance into the human living environment inevitably without consideration of whether these products are suitable and necessary for humans or not. As a countermeasure, LOHAS (Lifestyles of Health and Sustainability) attracts many people in the world now. In the same way, the authors think that manufacturing and design based on physiological polymorphism may become one of the most effective keys to keeping the optimum living environment for human beings.

Although the discovery and elucidation of physiological polymorphism are gradually advancing in the field of physiological anthropology, these findings have not been systematized yet enough to give feedback to manufacturing. Therefore, there is little concrete methodology to apply physiological polymorphism to manufacturing. For example, Inoue (2005), one of the authors, produced the following plan as a draft proposal.

A certain physiological response provoked by a certain stimulus (or stimuli) is evaluated by the measure $F$. A schematic histogram of $F$ is shown in Fig. 1a. Conventional manufacturing has been performed based on the value near the mean or mode of this distribution. If the manufacturers take into account individual differences, they may try to extend the coverage (the two-directional arrow in Fig. 1a) to the large side of the distribution. This is so-called universal design.

Here if someone suspects that this distribution is composed of two parts, group A in the smaller $F$ and group B in the larger $F$, in other words, if someone notices the existence of physiological polymorphism in this physiological response (Fig. 1b), two optimum ranges for group $\mathrm{A}$ and $\mathrm{B}$ are applied, respectively. Based on this idea, the previous expression $\partial D / \partial t \cong 0$ will be extended as follows: $\partial D / \partial t<0$ or $\lim _{t=\infty} D=0$. The latter two formulae mean that stress decreases as time goes by, and lead to a more positive pursuit of the user's comfort (pleasantness). Though Inoue's proposal is only one of the concepts for manufacturing and design based on physiological polymorphism, this is something like a bridge between standardized mass-production and custom-made adjustments for individuals. 

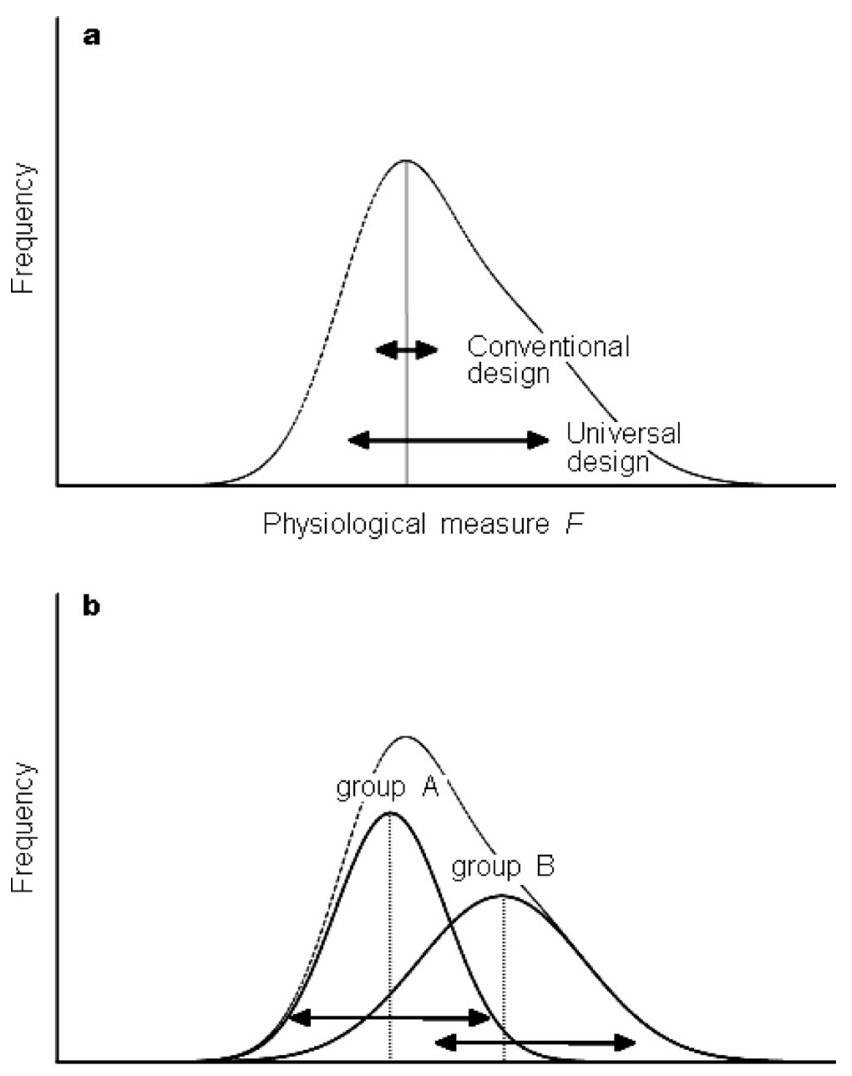

Physiological measure $F$

Fig. 1 Schematic distribution of physiological measures. The twodirectional arrows show the coverage of the manufacturing or design.

The authors have discussed repeatedly the target and the methodology for this new manufacturing based on each one's forte, that is, welfare equipment, clothes, artificial tissue, sporting gear, furniture, building materials, and human interface. The points of the discussion about each item are as follows.

Welfare equipment: Because physiological polymorphism arises in the process of genetic adaptation and environmental adaptation, elderly people who have been exposed to various environments for a long period express wide polymorphism psychologically and physically. A good example is the 70-yearold who reached the top of Mt. Everest while there are bedridden elderly who are the same age. Therefore it is almost impossible to design certain types of welfare equipment equally suitable for every elderly person, and to design individual equipment for each elderly person is also very difficult. Though universal design is one of the effective options we have to develop welfare equipment, this concept also tries to cover all elderly people by means of one single design. Detecting physiological polymorphism in the elderly may be a good way to develop good welfare equipment optimized to every physiological type, though it is not custommade.

Clothes: The thermal sensations of humans are fairly different from each other. Even if people stay in the same thermal environment, some people may feel hot, others may feel cool, and the rest may feel neither. Due to this, it is very difficult to standardize comfortable clothes which are suitable for the human living environment. If the variation of the thermal sensation is elucidated in relation to physiological polymorphism, a rational standard to produce the comfort clothes can be established. For instance, an elderly person's threshold of skin warm-cold sensitivity is higher than a young person's, and the individual difference is large. The individual differences in thermoregulatory response and body-form functionality of elderly people are also large. In the design of clothes for the elderly, it is very important to consider how to handle individual difference appropriately.

Artificial tissue: Artificial tissue must take over the function of living tissue. Because a disorder in the local biological regulation mechanism maintained by a certain tissue might cause an undesirable change to the whole body, polymorphism at the cell-level has to be considered in the design of artificial tissue. For example, a blood vessel changes its shape according to the stimulation which is caused by the shear stress between the blood flow and the vessel wall. The verification of the polymorphism in this reaction is very important in the development of artificial blood vessels.

Sporting gear: In order to preserve and promote one's present state of health, a proper amount of regular exercise is recommended. As if responding to this recommendation, various sporting gear has been developed, and many urban dwellers have fun in various sports by using these products. Many users of such sporting gear are amateurs. The choice of an appropriate sporting product is very important so that every amateur user can enjoy sports safely and comfortably without hesitation over their age and sex. However, the physical conditions and/or physical abilities of the users vary widely. It is virtually impossible to optimize any sporting gear for all amateurs, though it is possible to adjust it for the some major athletes. If the concept of physiological polymorphism is introduced to the design of sporting gear, this dilemma will be solved to some extent. Physiological measurement is performed on humans who do a certain physical exercise with a certain sporting product. At the same time, a lot of past physiological data concerning this exercise are scrutinized from the viewpoint of physiological polymorphism. By this means, if users are classified into several groups that show different physiological responses from each other, the development of physiologically suitable sporting gear for each user will become easier.

Furniture: Furniture is one of the indispensable tools for humans to live a cultural life or perform some sort of productive activity. A chair is one of the most common items of furniture that people use very frequently in daily life. Some users may sit on the chair almost all day long as a result of their work or body condition. Development of a chair which accords with each user will directly improve a user's QOL. For the chair design it will be very important to investigate how the hardness, thermal conductivity, moisture permeability, and so 
on, of the seat influences the physiological responses of the users, and to check whether physiological polymorphism exists or not. Furthermore, to clarify the difference of physiological responses of users who touch the various materials (wood and steel, for example) used in the chair is very important in decision-making regarding product development, or the selection of an appropriate sales policy.

Building materials: The human living environment consists of various building materials. In particular, material of natural origin, like wood, often has its high affinity with humans pointed out. Even if an urban high-rise condominium is constructed of iron and concrete, a large amount of wood is used in its interior, which is directly seen and touched by residents. It is very probable that the low thermal conductivity, moderate surface roughness and rigidity, warm color, fluctuating pattern, aroma, and so on, of these materials contribute to its high degree of affinity with humans as stimuli to provoke various physiological responses. However, there has been no systematic examination of how widely these responses vary as a result of individual difference. A survey about not only the physiological effect of building materials but the variety of the physiological response is very important because urbanized people tend to spend a longer time in closed artificial space. In order to develop new building materials more physiologically suitable for the human, a database which contains information about the various properties of the materials and the physiological responses of humans provoked by these materials should be prepared. If this database supports a special search from the viewpoint of physiological polymorphism, selection of the building materials for a living space would be carried out more carefully to adapt to each resident.

Human interface: Various machinery and tools (home electronics, information devices, industrial machines, and so on) operated by people are equipped with human interfaces. When such a human interface runs, a user will be urged to enter various strings of input to the interface, and the user will be requested to receive various sorts of output from the interface. At this time, the user will suffer a physiological load to some extent. The most desirable interface demands only a small physiological load of the users while offering them high performance. However, the design of an interface that pays attention to physiological load quantitatively is rare. Thereupon an interface designer will investigate the task performance of the users who operate the prototype while recording their evoked potential, EEG, electromyogram, cardiovascular response, electrodermal response, and so on. Based on this data the interface designer will evaluate the prototype and try to produce the optimum interface. At that time, the variation that originated from physiological polymorphism may be reflected in the data. The designers should consider this point when they design a better human interface.

\section{Future Prospects}

In conclusion, on the basis of the above discussion, the procedure that will be needed to achieve manufacturing and design based on physiological polymorphism is proposed here.

1) When the design of a product is started, it is necessary to specify the physiological measurement that should be considered at the beginning. Whether significant polymorphism is detected in the assumed user group is examined from the various prior studies or new experiments. If physiological polymorphism is revealed here, the user group is classified by using the concept of Fig. 1b, and the design of the product is optimized to each group.

2) Even if polymorphism is not confirmed in the above survey or experiments, the possibility that polymorphism might be revealed in the critical-state is not denied. In this case, it is necessary to set the range where polymorphism does not usually appear, and to optimize the design within this range. In addition, a design with physiological adjustability should be examined in consideration of the use of a more wide-ranging user. Though this concept might seem to be like the universal design shown in Fig. 1a, this concept is quite different from the universal design because it defines the target range clearly, based on physiological measures and also including physiological adjustability.

Acknowledgments This work was supported by a Grantin-Aid for Scientific Research (No.16637007) from the Japan Society for the Promotion of Science.

\section{References}

Iguchi S (1994) What is Kansei information? In Iguchi A ed. Kansei information processing. Ohm-sha, Tokyo, 3-6 [In Japanese]

Inoue K (2005) Manufacturing of a Wheelchair based on Physiological Anthropology. The Background. The handout of the 1st meeting of the "Manufacturing research group." [In Japanese]

Iwanaga K, Liu X, Shimomura Y, Katsuura T (2005) Approach to Human Adaptability to Stresses of City Life. J Physiol Anthropol Appl Human Sci 24: 357-361

Maeda T (2005) Perspectives on Environmental Adaptability and Physiological Polymorphism in Thermoregulation. J Physiol Anthropol Appl Human Sci 24: 237-240

Miyazaki Y, Tsunetsugu Y (2005) A Tentative Proposal on Physiological Polymorphism and Its Experimental Approaches. J Physiol Anthropol Appl Human Sci 24: 297-300

Sato M (2005) The Development of Conceptual Framework in Physiological Anthropology. J Physiol Anthropol Appl Human Sci 24: 289-295

Shimizu Y (1996) Sensitivity and Kansei engineering. In Shinohara A, Sakamoto H, Shimizu Y eds. Invitation to Kansei engineering. Morikita Shuppan, Tokyo, 12-17 [In 


\section{Japanese]}

Takagi Y, Harada H, Maeda T, Sato M (2006) Physiological Anthropology Design: A Comparative Study between Germany and Japan. J Physiol Anthropol 25: 55-57

Yasukouchi A (2005) A Perspective on the Diversity of Human Adaptability. J Physiol Anthropol Appl Human Sci 24: 243247
Received: December 4, 2006

Accepted: April 20, 2007

Correspondence to: Masashi Nakamura, Division of Forest and Biomaterials Science, Graduate School of Agriculture, Kyoto University, Kitashirakawa Oiwake-cho, Sakyo-ku, Kyoto 6068502, Japan

Phone: +81-75-753-6237

Fax: +81-75-753-6300

e-mail: nakamasa@kais.kyoto-u.ac.jp 